\title{
Editorial
}

\section{Is complex regional pain syndrome an inflammatory process? Theories and therapeutic implications}

\author{
Rame Taha MD, Gilbert Blaise MD
}

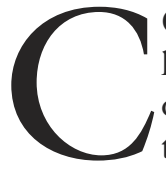
OMPLEX regional pain syndrome (CRPS) has an unknown etiology. It is a painful disorder that develops mostly as a disproportionate consequence of trauma to a joint or limb without nerve injury (CRPS I) or with obvious nerve lesions (CRPS II). Complex regional pain syndrome is a relatively common disabling disorder and a clinically challenging entity, both in terms of accurate diagnosis and effective treatment. Complex regional pain syndrome is characterized clinically by sensory, autonomic and motor disturbances. ${ }^{1}$

Various theories about disease mechanisms related to CRPS have been published, but many questions remain unresolved. However, over the past decade, an increasing number of studies have provided insights into the neuro-inflammatory mechanisms that underlie the pain disorders of CRPS. It is clear that inflammation plays a significant role in the pathogenesis of several spinal disorders, such as disc herniation and sciatica, which have previously been regarded as being primarily mechanical or degenerative in nature. ${ }^{2}$ Clinical features of acute CRPS (vasodilatation, swelling, local temperature changes, pain) indicate a localized inflammatory process. New data support an exaggerated regional inflammatory response as the pathophysiological mechanism underlying CRPS. Interleukine-6 (IL-6) and tumour necrosis factoralpha (TNF- $\alpha$ ) in blister fluid of the affected extremities of CRPS patients are significantly higher than in the uninvolved extremity, and these higher levels correlate significantly with disease activity and impairment. ${ }^{3}$ Alexander et al. ${ }^{4}$ have demonstrated a significant increase of IL- $1 \alpha$ and IL- 6 in the cerebrospinal fluid (CSF) of CRPS patients as compared to control subjects. In contrast, Schinkel et al. ${ }^{5}$ showed that IL-
8 and soluble TNF receptor $1 / \mathrm{II}$, but not IL-6, were significantly elevated in the venous blood of CRPS patients. Sample differences may explain the discrepancies in IL-6 level in these reports.

It has been well-documented that pro-inflammatory cytokines are involved in stimulating dorsal root ganglia to release substance $P$ and calcitonin generelated peptide (CGRP) in nerve injury diseases. ${ }^{6}$ Previous data have demonstrated elevated CGRP and substance P levels in serum samples from patients with acute CRPS, and that neurogenic inflammation contributes to the clinical manifestations of acute CRPS. In contrast, more recent data from Albrecht et al. ${ }^{7}$ have shown that CGRP-positive innervation is significantly decreased compared with control skin from chronic CRPS patients. This discrepancy could be due to variances in disease stages or patient characteristics. It has been well indicated that pain and hyperalgesia, particularly in the chronic stage, are independent of increased neuropeptide concentration, indicating that neurogenic inflammation alone is unable to explain the occurrence of allodynia, hyperalgesia, and movement disorders in patients with CRPS. These features of CRPS are most likely manifestations of central sensitization, a mechanism in which spinal circuits involved in sensory motor integration become impaired, despite unchanged afferent input. Central sensitization is the pivotal physiological phenomenon underlying centrally mediated exaggerated pain states following an insult.

To a lesser extent, other mechanisms, such as oxidative stress, may play a significant role in the pathophysiology of CRPS. Many pain-related diseases e.g., fibromyalgia, Raynaud's disease and peripheral neuropathy ${ }^{8}$ are associated with oxidative stress phe-

CAN J ANESTH $2007 / 54: 4 /$ pp 249-253

From the Laboratory of Anesthesia, Department of Anesthesia, Centre hospitalier de l'Université de Montréal (CHUM) - Hôpital Notre-Dame, Montréal, Québec, Canada.

Address correspondence to: Dr. Gilbert Blaise, Laboratory of Anesthesia, Department of Anesthesia, Centre hospitalier de l'Université de Montréal (CHUM) - Hôpital Notre-Dame, 1560, rue Sherbrooke est, Montréal, Québec H2L 4M1, Canada. Phone: 514-890-8000, ext. 25397; Fax: 514-412-7520; E-mail:blaisegil@videotron.ca 
nomena. Oxygen consumption is decreased in limbs affected by CRPS, and pain reduction following treatment with oral vasodilators has been demonstrated. ${ }^{9}$

Nitric oxide (NO), a small gaseous free radical molecule, demonstrates an interesting anti-inflammatory profile and vascular protective activity. ${ }^{10}$ High NO levels have been documented in CRPS patients. Stimulation of peripheral blood monocytes in CRPS patients by interferon-gamma leads to increase NO production. ${ }^{11}$ Shin $e t$ al. have reported that inhibition of inducible NO synthase promotes motor function recovery in rats after sciatic nerve ischemia and reperfusion. ${ }^{12}$ The highly beneficial effect of bioavailable $\mathrm{NO}$ is attributed to its ability to scavenge superoxide, the main component of oxidative stress. The use of inhaled NO in the early stage of the disease might open a new era of CRPS research.

Although there are no clearly delineated stages in the evolution of CRPS, we believe that the inflammatory process begins at an early, mild stage, then becomes progressive as the inflammation plays a pivotal role in sustaining the pain. The mechanisms and targets of treatment are probably different in early and late stages of the disease.

\section{Complex regional pain syndrome and research models}

Complex regional pain syndrome research will remain in a vicious circle unless an animal model is developed that helps researchers to identify the mechanisms of pain production, and the associated changes in sensory and motor function. Difficulties in examining the pathology have complicated our understanding of the mechanisms underlying this syndrome. The following issues need to be clarified:

1. What is the best tissue site (local, or remote from the disease process) to examine? Is it best to obtain control specimens from the same patient or healthy control subjects?

2. What are the optimal tissue markers (blood, CSF, blister fluid, skin biopsies, muscle biopsies) to investigate?

3. Are there differences in the mechanisms underlying acute and chronic conditions? Should inflammatory mediators be compared in the acute and chronic stages of the disease process?

\section{Therapeutic implications}

A major limitation in identifying treatment strategies for patients with CRPS is that the syndrome represents a spectrum of heterogeneous clinical manifestations. Numerous therapeutic modalities have been applied, often with limited success. Non-steroidal anti-inflam- matory drugs, opioid analgesics, targeted injections of local anesthetics to block pain fibres in sympathetic nerves, and transcutaneous electrical nerve stimulation have all produced variable results.

Therapeutic approaches involving cytokine inhibition have been tested experimentally, and are already being developed in preliminary clinical trials in immune-mediated diseases. Immunomodulator therapy can inhibit the secretion of pro-inflammatory cytokines, increase the secretion of the anti-inflammatory cytokine, and induce T-cell proliferation. To date, gene therapy for pain control has targeted neurons. However, recent evidence indicates that spinal cord glia is critical to the creation and maintenance of pain facilitation through the release of pro-inflammatory cytokines. In an animal model of pain, Milligan et $a l .{ }^{13}$ have demonstrated that administration of rat IL10 protein itself briefly reversed chronic constriction injury-induced mechanical allodynia, thermal hyperalgesia and pain facilitation. Huygen $e t a l$. have reported successful treatment of CRPS I with anti-TNF- $\alpha$. Antibodies against TNF- $\alpha$ are now in clinical use for the treatment of arthritis, and are proving to be very successful both in treating disease symptoms, including pain, and in modifying the course of the disease. To date, no study has targeted the CGRP levels in acute or chronic CRPS.

Recent work has demonstrated that chemokines and their receptors are also expressed in the nervous system. Intradermal application of several chemokines produces a strong pain response. Drugs that block chemokine receptors, such as CCR-2, might constitute a novel class of therapeutic agents for the treatment of painful sensory neuropathies. Free radical scavengers, $\mathrm{N}$-acetyl-L-cysteine and 4-hydroxy-2,2,6,6-tetramethylpiperidine, reduce signs of hyperalgesia and allodynia in animal models of CRPS. ${ }^{14}$ Clinically, topical treatment with $50 \%$ dimethyl sulfoxide cream can be effective in decreasing the hypoxia-related production of free oxygen radicals. ${ }^{15}$ In CRPS type 1, post-stroke upper extremity hemiplegia may be prevented by early inpatient rehabilitation and avoidance of shoulder trauma to the affected arm.

\section{Future directions}

Current best evidence indicates that the mechanisms underlying CRPS involve different biological pathways which include inflammation (local, systemic), central processing of afferent input, and oxidative stress. The presence of pro-inflammatory cytokines in serum, blister fluid, CSF and many inflammatory cells, such as activated $\mathrm{T}$ lymphocytes, monocytes, and skin resident cells like mast cells, support the hypothesis that CRPS 
is part of a systemic inflammatory process. To further our understanding of the complex inter-related disease mechanisms and to develop optimal treatment strategies, we must identify the critical points in our basic knowledge of CRPS, as well as potential directions for basic and clinical research on new treatment strategies. An effective way to achieve this overall goal is to develop targeted bench-to-bedside research programs in association with chronic pain clinics in which CRPS patients are diagnosed and treated.

\section{Le syndrome dou- loureux régional complexe est-il un pro- cessus inflammatoire? Théories et implications thérapeutiques}

L'étiologie du syndrome douloureux régional complexe (SDRC) est inconnue. Il s'agit de troubles douloureux qui se développent la plupart du temps en tant que conséquences disproportionnées d'un traumatisme à une articulation ou à un membre sans blessure neuronale (SDRC I), ou avec des lésions neuronales évidentes (SDRC II). Le syndrome douloureux régional complexe est un trouble handicapant relativement fréquent ; c'est également une entité posant des défis cliniques au niveau de la précision du diagnostic et du traitement efficace. Le syndrome douloureux régional complexe se caractérise cliniquement par des désordres sensitifs, autonomes et moteurs. ${ }^{1}$

Plusieurs théories traitant des mécanismes pathologiques liés au SDRC ont été publiées, mais de nombreuses questions sont encore sans réponse. Néanmoins, ces dix dernières années, un nombre croissant d'études ont fourni un aperçu des mécanismes neuro-inflammatoires qui sous-tendent les troubles douloureux liés au SDRC. L'inflammation joue clairement un rôle majeur dans la pathogénie de nombreux troubles rachidiens tels que hernie discale et sciatique, deux troubles précédemment considérés comme principalement de nature mécanique ou dégénérative. ${ }^{2}$ Les traits cliniques d'un SDRC aigu (vasodilatation, gonflement, changements de température locale, douleur) indiquent un processus d'inflammation localisée. Une réaction régionale inflammatoire exagérée est considérée responsable du mécanisme physiopathologique sous-jacent au SDRC par de nouvelles données. Dans les extrémités affectées des patients à SDRC, les taux d'interleukine-6 (IL-6) et de facteur nécrosant des tumeurs alpha (TNF$\alpha$ ) sont significativement plus élevés dans le liquide d'ampoules que dans les extrémités non touchées, et ces niveaux plus élevés sont en haute corrélation avec l'activité de la maladie et la déficience. ${ }^{3}$ Alexander et coll. ${ }^{4}$ ont démontré une augmentation significative de IL- $1 \alpha$ et de IL- 6 dans le liquide céphalo-rachidien (LCR) des patients à SDRC par rapport à des sujets témoins. En revanche, Schinkel et coll. ${ }^{5}$ ont montré que le IL-8 ainsi que le récepteur TNF 1/II, mais non pas le IL-6, étaient significativement plus élevés dans le sang artériel de patients à SDRC. Des différences d'échantillons pourraient expliquer les écarts dans les taux de IL-6 de ces deux études.

Il a été clairement établi que les cytokines proinflammatoires jouent un rôle dans la stimulation des ganglions de la racine postérieure qui libèrent de la substance $\mathrm{P}$ et du peptide lié au gène de la calcitonine (CGRP) dans les maladies de blessures neuronales. ${ }^{6}$ Des données antérieures ont démontré des taux élevés de CGRP et de substance $\mathrm{P}$ dans des échantillons de sérum de patients souffrant de SDRC aigu, et que l'inflammation neurogène contribue aux manifestations cliniques de SDRC aigu. En revanche, des données plus récentes de Albrecht et coll. ${ }^{7}$ ont montré que l'innervation positive contenant du CGRP est significativement plus basse comparée à de la peau-témoin de patients de SDRC chronique. Cette différence peut être expliquée par une variation dans les étapes de la maladie ou dans les caractéristiques des patients. Il a été clairement indiqué que la douleur et l'hyperalgésie, particulièrement dans la phase chronique du SDRC, sont indépendantes d'une concentration accrue de neuropeptides, indiquant que l'inflammation neurogène seule ne peut expliquer l'apparition d'allodynie, d'hyperalgésie et de troubles du mouvement chez les patients souffrant de SDRC. Ces particularités du SDRC sont probablement des manifestations d'une sensibilisation centrale, un mécanisme par lequel les circuits rachidiens impliqués dans l'intégration sensori-motrice sont affaiblis malgré un trafic afférent inchangé. La sensibilisation centrale est le phénomène physiologique clé qui sous-tend les états de douleur exagérée gérés de façon centrale suite à une blessure.

Dans une moindre mesure, d'autres mécanismes tels que le stress oxydatif peuvent jouer un rôle 
important dans la physiopathologie du SDRC. De nombreuses maladies liées à la douleur, par ex. la fibromyalgie, la maladie de Raynaud et la neuropathie périphérique ${ }^{8}$ sont associées aux phénomènes de stress oxydatif. La consommation d'oxygène diminue dans les membres atteints de SDRC, et une réduction de la douleur suite à un traitement avec des vasodilatateurs oraux a été démontrée. ${ }^{9}$

L'oxyde nitrique (NO), une petite molécule gazeuse et radicale libre, présente un profil anti-inflammatoire intéressant et une activité vasculaire protectrice. ${ }^{10}$ Des taux élevés de NO ont été remarqués chez des patients souffrant de SDRC. La stimulation par interféron gamma des monocytes sanguins périphériques chez les patients souffrant de SDRC provoque une production accrue de NO. ${ }^{11}$ L'étude de Shin et coll. montre que l'inhibition de NO synthase inductible encourage le rétablissement de la fonction motrice chez les rats après une ischémie du nerf sciatique et une reperfusion. ${ }^{12}$ L'effet hautement bénéfique du NO biodisponible est attribué à sa capacité à piéger le superoxyde, le composant principal du stress oxydatif. L'utilisation du NO inhalé durant la première phase de la maladie pourrait ouvrir de nouvelles voies à la recherche sur le SDRC.

Bien qu'il n'existe pas de phases clairement délimitées dans l'évolution du SDRC, nous pensons que le processus inflammatoire commence à un stade précoce et léger, puis devient progressif tandis que l'inflammation joue un rôle clé dans le maintien de la douleur. Les mécanismes et les cibles de traitement sont vraisemblablement différents dans les différentes phases de la maladie.

\section{Le syndrome douloureux régional complexe et les modèles de recherche}

La recherche sur le syndrome douloureux régional complexe est condamnée à demeurer dans un cercle vicieux tant qu'un modèle animal n'aura pas été développé afin d'aider les chercheurs à identifier les mécanismes de production de la douleur ainsi que les changements dans la fonction sensori-motrice qui y sont liés. Des difficultés rencontrées lors de l'étude de la pathologie ont compliqué notre compréhension des mécanismes qui sous-tendent ce syndrome. Les questions suivantes doivent être clarifiées :

1. Quel est le meilleur site (local, ou éloigné du processus de maladie) à examiner ? Est-il mieux d'obtenir des spécimens témoins des mêmes patients ou de sujets témoins sains ?

2. Quels sont les marqueurs tissulaires optimaux (sang, FCS, liquide de l'ampoule, biopsies de la peau, biopsies de muscles) pour la recherche ?
3. Existe-t-il des différences dans les mécanismes sous-jacents aux conditions aiguës et chroniques ? Les médiateurs inflammatoires devraientils être comparés dans les phases aiguës et chroniques du processus de maladie ?

\section{Implications thérapeutiques}

Une des limites majeures dans l'identification de stratégies de soins pour les patients souffrant de SDRC est le fait que le syndrome se présente comme une gamme de manifestations cliniques hétérogènes. De nombreuses modalités thérapeutiques ont été utilisées, souvent avec un succès limité. Des résultats variables ont été produits par toutes les modalités thérapeutiques suivantes: médicaments anti-inflammatoires non-stéroïdiens, analgésiques opiacés, injections ciblées d'anesthésiques locaux pour bloquer les fibres de la douleur dans les nerfs sympathiques, et stimulation nerveuse électrique transcutanée.

Des approches thérapeutiques se servant de l'inhibition de cytokine ont été testées expérimentalement et sont d'ores et déjà développées dans des études cliniques préliminaires sur des maladies immunitaires. Une thérapie immunomodulatrice peut inhiber la sécrétion de cytokine pro-inflammatoire, augmenter la sécrétion de cytokine anti-inflammatoire, et provoquer la prolifération de cellules T. À ce jour, la thérapie génique pour le contrôle de la douleur a pour cible les neurones. Toutefois, des données probantes récentes indiquent que les cellules gliales de la moelle épinière sont essentielles à la création et au maintien de la facilitation de la douleur par la libération de cytokines pro-inflammatoires. Dans un modèle de douleur animal, Milligan et coll. ${ }^{13}$ ont montré que l'administration de protéine IL-10 de rat a brièvement inversé l'allodynie mécanique provoquée par une blessure de constriction chronique, l'hyperalgésie thermique et la facilitation de la douleur. Dans leur étude, Huygen et coll. font état du traitement réussi de SDRC I avec des anti-TNF- $\alpha$. Les anticorps antiTNF- $\alpha$ sont désormais utilisés en pratique clinique comme traitement de l'arthrite, et ils s'avèrent très performants dans le traitement des symptômes de la maladie, y compris la douleur, et dans la modification de l'évolution de la maladie. A ce jour, aucune étude n'a examiné les taux de CGRP dans les cas de SDRC aigus ou chroniques.

Des études récentes ont montré que les chimiokines ainsi que leurs récepteurs sont également exprimés dans le système nerveux. L'application intradermique de plusieurs chimiokines produit une forte réaction de douleur. Les médicaments bloquant les récepteurs de chimiokines, tels que le CCR-2, pourraient constituer 
une nouvelle catégorie d'agents thérapeutiques pour le traitement de neuropathies sensitives douloureuses. Les piégeurs de radicaux libres - $\mathrm{N}$-acetyl-L-cystéine et 4-hydroxy-2,2,6,6-tetramethylpiperidine - réduisent les signes d'hyperalgésie et d'allodynie dans les modèles animaux de SDRC. ${ }^{14}$ Dans la pratique clinique, le traitement topique avec de la crème de sulfoxyde de dimethyl $50 \%$ peut s'avérer efficace pour réduire la production de radicaux d'oxygène libres liée à l'hypoxie. ${ }^{15}$ Dans le SDRC de type 1, l'hémiplégie des membres supérieurs post-attaque peut être évitée par la réhabilitation précoce en milieu hospitalier et par le fait d'éviter un traumatisme à l'épaule du bras atteint.

\section{Orientations futures}

Les meilleures données probantes actuelles indiquent que les mécanismes sous-jacents au SDRC impliquent différents circuits biologiques, qui comprennent l'inflammation (locale, systémique), la gestion centrale des afférences et le stress oxydatif. La présence de cytokines pro-inflammatoires dans le sérum, le liquide de la lésion, le LCR et de nombreuses cellules inflammatoires telles que les lymphocytes $\mathrm{T}$ activés, les monocytes et les cellules résidentes de la peau comme les mastocytes, appuient l'hypothèse que le SCRC fait partie d'un processus inflammatoire systémique. Afin de faire progresser notre compréhension des mécanismes complexes étroitement liés de la maladie et de développer des stratégies de traitement optimales, nous devons identifier les points critiques dans notre connaissance de base du SCRC ainsi que des directions potentielles pour la recherche fondamentale et clinique de nouveaux traitements. Un bon moyen d'atteindre ce but global est de développer des programmes de recherches ciblés 'du laboratoire au patient' en coordination avec les cliniques de la douleur chronique où les patients souffrant du SDRC sont diagnostiqués et soignés.

\section{References}

1 Harden RN. Complex regional pain syndrome. Br J Anaesth 2001: 87: 99-106.

2 Takabashi N, Kikuchi S, Shubayev VI, Campana WM, Myers RR. TNF-alpha and phosphorylation of ERK in DRG and spinal cord: insights into mechanisms of sciatica. Spine 2006; 31: 523-9.

3 Huygen FJ, De Bruijn AG, De Bruin MT, Groeneweg $J G$, Klein J, Zijistra FJ. Evidence for local inflammation in complex regional pain syndrome type 1. Mediators Inflamm 2002; 11: 47-51.

4 Alexander GM, van Rijn MA, van Hilten JJ, Perreault $M J$, Schwartzman RJ. Changes in cerebrospinal fluid levels of pro-inflammatory cytokines in CRPS. Pain
2005; 116: 213-9.

5 Schinkel C, Gaertner A, Zaspel J, Zedler S, Faist E, Schuermann $M$. Inflammatory mediators are altered in the acute phase of posttraumatic complex regional pain syndrome. Clin J Pain 2006; 22: 235-9.

6 Weber M, Birklein F, Neundorfer B, Schmelz M. Facilitated neurogenic inflammation in complex regional pain syndrome. Pain 2000; 91: 251-7.

7 Albrecht PJ, Hines S, Eisenberg E, et al. Pathologic alterations of cutaneous innervation and vasculature in affected limbs from patients with complex regional pain syndrome. Pain 2006; 120: 244-66.

8 Bagis S, Tamer L, Sabin G, et al. Free radicals and antioxidants in primary fibromyalgia: an oxidative stress disorder? Rheumatol Int 2005; 3: 188-90.

9 Goris RJ. Reflex sympathetic dystrophy: model of a severe regional inflammatory response syndrome. World J Surg 1998; 22: 197-202.

10 Blaise GA, Gauvin D, Gangal M, Authier S. Nitric oxide, cell signaling and cell death. Toxicology 2005; 208: 177-92.

11 Hartrick CT. Increased production of nitric oxide stimulated by interferon-gamma from peripheral blood monocytes in patients with complex regional pain syndrome. Neurosci Lett 2002; 323: 75-7.

12 Shin SJ, Qi WN, Cai $\Upsilon$, et al. Inhibition of inducible nitric oxide synthase promotes recovery of motor function in rats after sciatic nerve ischemia and reperfusion. J Hand Surg 2005; 30: 826-35.

13 Milligan ED, Langer SJ, Sloane EM, et al. Controlling pathological pain by adenovirally driven spinal production of the anti-inflammatory cytokine, interleukin-10. Eur J Neurosci 2005; 21 : 2136-48.

14 Xanthos D, Francis L, Bennett G, Coderre T. Animal models of chronic pain: chronic post-ischemia pain: a novel animal model of complex regional pain syndrome type I produced by prolonged hindpaw ischemia and reperfusion in the rat. J Pain 2004; 5(3 Suppl 2): S1.

15 Zuurmond WW, Langendijk PN, Bezemer PD, Brink $H E$, de Lange JJ, van loenen AC. Treatment of acute reflex sympathetic dystrophy with DMSO 50\% in a fatty cream. Acta Anaesthesiol Scand 1996; 40: 364-7. 